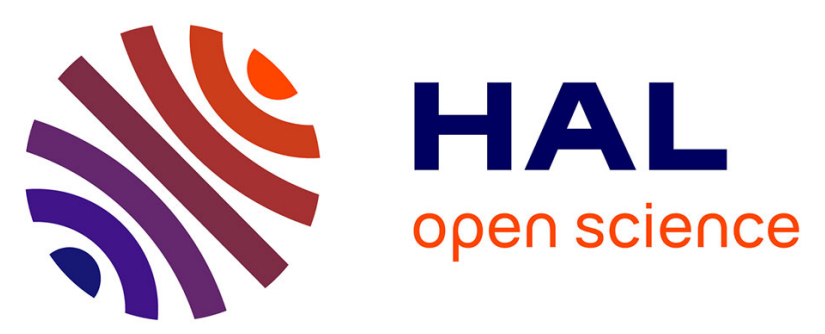

\title{
Un outil de GTEC : la mise en place d'une charte de l'emploi saisonnier dans le secteur du végétal spécialisé.
}

Stéphane Fauvy, Nicolas Arnaud

\section{To cite this version:}

Stéphane Fauvy, Nicolas Arnaud. Un outil de GTEC: la mise en place d'une charte de l'emploi saisonnier dans le secteur du végétal spécialisé. : Le cas des rosiéristes du Douessin. Revue management \& avenir, 2012, 56, pp.78-98. 10.3917/mav.056.0054 . hal-00837589

\section{HAL Id: hal-00837589}

\section{https://hal-audencia.archives-ouvertes.fr/hal-00837589}

Submitted on 23 Jun 2013

HAL is a multi-disciplinary open access archive for the deposit and dissemination of scientific research documents, whether they are published or not. The documents may come from teaching and research institutions in France or abroad, or from public or private research centers.
L'archive ouverte pluridisciplinaire HAL, est destinée au dépôt et à la diffusion de documents scientifiques de niveau recherche, publiés ou non, émanant des établissements d'enseignement et de recherche français ou étrangers, des laboratoires publics ou privés. 


\title{
Un outil de GTEC : la mise en place d'une charte de l'emploi saisonnier dans le secteur du végétal spécialisé. Le cas des rosiéristes du Douessin ${ }^{1}$.
}

\author{
Stéphane FAUVY,
}

ESSCA Ecole de Management, LUNAM Université

stephane.fauvy@essca.fr

\section{Nicolas ARNAUD}

Audencia Nantes Ecole de Management, GREMA

nicolasarnaud@audencia.com

\section{Résumé}

Cet article s'intéresse au développement et à la mise en place d'une charte de l'emploi saisonnier au sein du secteur du végétal spécialisé. Cette charte constitue aux yeux des acteurs impliqués dans ce projet un outil emprunt d'une philosophie gestionnaire à n'en pas douter « bienveillante et durable », visant à diffuser auprès des entreprises du secteur ayant massivement recours à de l'emploi saisonnier de bonnes pratiques en matière de gestion des ressources humaines. L'enquête menée par les auteurs a permis de mettre en évidence un décalage entre les intentions de l'outil et les problématiques plus profondes rencontrées par les rosiéristes du Douessin, premiers producteurs de roses en France.

\section{Mots-clés}

GTEC, territoire, emploi saisonnier, secteur agricole

\begin{abstract}
This paper focuses on the development and implementation of a charter of seasonal employment in the sector of specialized plants. This charter constitutes in the eyes of those involved in this project a tool borrowing from a managerial philosophy based on "care and responsibility", aiming to disseminate good practices in human resources management within companies using seasonal employees. The survey conducted has highlighted a gap between the intentions of the tool and the deeper issues faced by the first French roses growers of the Douessin area.
\end{abstract}

\section{Key words}

Territorial HRM, territory, seasonal, agriculture

\footnotetext{
${ }^{1}$ Cet article a fait l'objet d'une communication au XXII ${ }^{\circ}$ congrès de l'AGRH 2011, Marackech, Maroc.
} 


\section{Introduction}

Cet article s'intéresse au développement et à la mise en place d'une charte de l'emploi saisonnier au sein du secteur du végétal spécialisé. Cette charte constitue aux yeux des acteurs impliqués dans ce projet un outil empreint d'une philosophie gestionnaire à n'en pas douter «bienveillante et durable », visant à diffuser auprès des entreprises d'un secteur ayant massivement recours à de l'emploi saisonnier de bonnes pratiques en matière de gestion des ressources humaines (cf. annexe 1).

L'enjeu est de taille. En effet, le secteur horticole, à l'instar du secteur agricole auquel il se rattache, se caractérise depuis plusieurs années par une pénurie de main d'œuvre sur le marché de l'emploi alors même que le niveau de chômage reste durablement élevé. Le Président de l'Association Départementale Emploi-Formation en Agriculture (ADEFA) présente la situation de la manière suivante : «Pour les entreprises concernées, les salariés saisonniers ont une valeur inestimable dont on a pu percevoir l'importance au cours des dernières années lorsqu'elle fait défaut. Cette valeur est fondée sur leur disponibilité à certains moments clés du cycle de production des entreprises. La contrepartie de cette valeur est alors de s'interroger sur l'itinéraire professionnel de ces travailleurs et sur le statut qui devrait leur assurer une stabilité et une perspective d'avenir ». Ainsi, au delà du caractère très local de cette recherche, la problématique de l'emploi et de la mutualisation de celui-ci constitue une réponse possible à la prise en compte des emplois saisonnier dans d'autres secteurs.

Une solution prometteuse consiste à élargir la réflexion en matière de gestion des ressources humaines au niveau du territoire (Calamel, et al. 2011, Colle, et al. 2009, Defélix, et al. 2007). C'est la raison pour laquelle cet article s'inscrit dans le champ de la Gestion Territoriale des Emplois et des Compétences (GTEC). La littérature s'accorde globalement autour des éléments suivant pour définir la GTEC : elle consiste en des pratiques de gestion des ressources humaines organisées (mutualisation des emplois et de la formation, mobilité régionales, GPEC territorialisée, etc.) à l'extérieur des frontières traditionnelles de la firme dans le but de renforcer la compétitivité d'un territoire géographique donné (Barabel, et al. 2009, Bories-Azeau et Loubes 2009, Bories-Azeau, et al. 2007, Mazzilli 2010). Les développements qui suivent ont pour objectif de répondre à la question de recherche suivante : dans quelle mesure un outil de GTEC peut-il améliorer l'attractivité et la fidélisation d'une main d'œuvre saisonnière?

Ce papier est structuré de la manière suivante. Dans un premier temps nous proposons une revue de la littérature afin de mettre en évidence les multiples formes des politiques de GTEC, répondant à des objectifs variés, fonction de contingences locales et/ou sectorielles. Cette première section sera également l'occasion de présenter des éléments importants relatifs au secteur agricole et à l'emploi saisonnier. La seconde partie du papier présente un outil de GTEC actuellement expérimenté au sein de quelques entreprises du secteur du végétal dans le but d'une meilleure prise en charge de l'emploi saisonnier. L'intérêt de cet outil est discuté à la lumière d'entretiens effectués avec des dirigeants d'exploitation horticole, des salariés saisonniers ainsi que des représentants institutionnels du territoire.

\section{Formes et objectifs des politiques de gestion territoriale des emplois et des compétences.}

\subsection{Les outils de la GTEC : des pratiques contingentes et contextualisées}

Les pratiques toujours plus nombreuses de gestion territoriale des emplois et des compétences (GTEC) revêtent, à l'instar des pratiques de GRH traditionnelles, des formes multiples répondant à des objectifs variés. A titre d'illustration, les mises à disposition de salariés, les pôles de mobilité régionaux, les 
plans concertés de gestion des compétences, les dispositifs de reclassement élargi, les parcours professionnels transverses constituent autant d'exemples de GTEC (Colle, et al. 2009, Defélix, et al. 2009, Defélix, et al. 2010, Mazzilli 2009). Ces pratiques sont par ailleurs portées par des structures diverses : groupements d'employeurs, comités de bassin d'emploi, branches professionnelles, Maisons de l'Emploi et de la Formation ou pôles de compétitivité (Bories-Azeau et Loubes 2009, Mazzilli 2010).

Quel que soit leur forme et leur institution d'origine, les pratiques de GTEC sont guidées par un objectif global de renforcement de la compétitivité des entreprises d'un territoire géographique donné. L'une des particularité des projets de GTEC réside dans le fait qu'ils cristallisent des acteurs qui "ne sont pas liés par des relations hiérarchiques, ni par des partages d'expérience ou de valeurs communes, [et qui] appartiennent généralement à des institutions différentes » (Raulet-Croset 2008, p.149) mais par des problématiques organisationnelles et stratégiques relativement proches nécessitant un regroupement et une mutualisation de la réflexion et des actions $\mathrm{RH}$, particulièrement dans cette recherche compte tenu de la petite taille des entreprises étudiées.

Si les pratiques territorialisées de GRH répondent globalement à des enjeux de compétitivité du territoire, les objectifs locaux sont multiples. En effet, s'il s'agit globalement d'assurer la compétitivité d'un territoire géographique donnée à l'heure de la mondialisation, les pratiques de GRH territoriales renvoient à des enjeux divers. Glee et Roger (2007) s'intéressent par exemple aux secteurs du batiment et de la restauration, secteurs reconnus pour des métiers peu valorisés. Dans leur enquête, ils montrent la difficulté qu'ont les entreprises de ces deux secteurs d'activité à attirer et fidéliser les salariés alors même que la France connaît un niveau de chômage structurel quasi inégalé dans son histoire. Le recours à de la main d'œuvre étrangère devient alors une solution de recours pour assurer la réalisation du volume d'activité.

Defelix et al. (2010) identifient par ailleurs dans le développement de la GTEC un levier de performance permettant de redynamiser un bassin d'emploi. Afin de faire face à la baisse de mobilité des salariés, les entreprises, souvent avec l'appui des collectivités territoriales, réfléchissent à de nouvelles possibilités locales permettant à la fois de fidéliser sur un territoire donné et de sécuriser les parcours de salariés les moins qualifiés à la recherche d'un emploi. Ainsi, on observe par exemple l'apparition de Plans de Mobilité Régionale (Mazzilli 2009).

Assurer la compétitivité des entreprises passe également par des actions visant à favoriser la circulation des connaissances au sein d'un secteur d'acteurs ou d'un réseau territorial. A titre d'exemple, Arnaud (2007) montrent dans sa thèse comment les dirigeants du leader européen de la prestation logistique du meuble ont largement recruté chez leurs clients (les fabricants de meubles et la grande distribution spécialisée) les cadres devant moderniser et industrialiser les pratiques et les processus de leur groupe. Cette pratique permet au prestataire de mieux comprendre les attentes afin de développer leur offre commerciale et organisationnelle tout en accédant à des modes de fonctionnement plus structurés et organisés. On notera avec Pichault (2010) que de telles pratiques seront d'autant plus efficaces qu'il existe un fort degré d'interdépendance entre les organisations. C'est la raison pour laquelle ces quelques exemples de GTEC, qu'elles soient ou non portées par des acteurs publics, ne doivent pas faire oublier que l'une des conditions de succès repose dans la capacité des acteurs "à créer de la proximité organisationnelle et institutionnelle dans un contexte de proximité géographique » (Zimmermann 2008).

\subsection{Secteur agricole, exploitation agricole et ressources humaines.}


Véritable activité professionnelle, avec ses alternances de phases d'activité et de périodes plus incertaines, le travail saisonnier s'est largement modifié au cours du temps. C'est la raison pour laquelle, après avoir brièvement dépeint l'évolution du monde rural et des entreprises agricoles, nous rendons compte de la diversité des profils des travailleurs saisonniers agricoles.

\subsubsection{Les entreprises agricoles : des structures diversifiées et évolutives}

Le secteur agricole n'échappe pas au processus de globalisation des économies et à l'impressionnante recomposition du monde paysan. En effet la globalisation de la production et des échanges agricoles a induit une forme de disqualification d'une grande partie des paysanneries du monde. La « fin des paysans » (Mendras 1970) s'est traduite par deux trajectoires qui demeurent d'actualité, à savoir le passage à la modernité et l'agriculture pour les uns, à l'exode pour les autres ${ }^{2}$.

En France, et plus généralement en Europe, l'évolution des entreprises agricoles est caractérisée par une bipolarisation des modes de production (Hervieu 2006). Cette bipolarisation rend compte de l'émergence d'exploitations de plus en plus concentrées et spécialisées qui assurent une large part de la production de matière brute agricole (sur 600.000 exploitations, 130.000 assurent $80 \%$ de la production). Parallèlement, nous trouvons des exploitations de petite taille, de plus en plus diversifiées combinant la production, la transformation et la commercialisation. Cette dynamique engendre des formes de relations au salariat qui sont très différentes. D'une manière générale, elle se traduit par une augmentation très nette du salariat dans le secteur agricole. Les études tendent effectivement à démontrer le passage de 600.000 à 1.000 .000 de salariés agricoles en 40 ans.

De plus, il est aisé de constater un brouillard sur l'ensemble du paysage et des statuts dans l'agriculture. A titre d'illustration, les formes sociétaires se sont multipliées. Outre les Sociétés Anonymes (SA) et les Sociétés Anonymes à Responsabilité Limitée (SARL), près de la moitié de la surface agricole française utile est détenue ou gérée par des Exploitations Agricoles à Responsabilité Limitée (EARL), des Sociétés Civiles d'Exploitation Agricole (SCEA) et des Groupements Agricoles d'Exploitation en Commun (GAEC). Si ces formes organisationnelles permettent de régler en partie la question de la transmission, ils ajoutent à la complexité des statuts pour les travailleurs agricoles.

Enfin, l'extrême morcellement des contrats de travail et la courte durée des contrats saisonniers sont deux caractéristiques importantes du secteur agricole. En France, sur un million de salariés agricoles, il existe 1.300.000 contrats de travail dont 200.000 contrats à durée indéterminée (Mutualité Sociale Agricole 2008). Cela signifie qu'il arrive fréquemment qu'une même personne ait plusieurs contrats. Face à cette situation, comment faire en sorte que les individus, et notamment les travailleurs saisonniers, puissent construire leur parcours professionnel et faire reconnaître leurs compétences ?

\subsubsection{Les travailleurs saisonniers}

Au sein de l'espace professionnel agricole, le groupe des salariés saisonniers revêt une très grande hétérogénéité. Longtemps considéré comme un «paysan sans terre » (Mendras 1970), le saisonnier est traditionnellement caractérisé par sa disponibilité et sa connaissance du milieu. Or les évolutions du secteur agricole sont marquées par le passage d'une activité domestique familiale à une activité professionnelle, pas toujours considérée valorisante et professionnalisante, ce qui n'est pas sans influence sur la composition de l'offre de main d'œuvre. Afin de parfaire notre connaissance de ce

\footnotetext{
${ }^{2}$ L'année 2008 marque de ce point de vue une année historique dans l'histoire de l'humanité dans la mesure où, pour la première fois, les populations urbaines sont plus importantes que les populations rurales (source : ONU, note $\mathrm{n}^{\circ} 6144,22$ avril 2008).
} 
groupe social qui n'en est pas vraiment un, l'étude de Guais (2007) est utile car elle relève quatre profils de saisonniers.

Les « vrais saisonniers » connaissent le fonctionnement des saisons de leur territoire et s'adaptent aux différentes saisonnalités proposées par les entreprises locales. Ils viennent tous les ans, sont inscrits dans une routine et passent d'une entreprise à une autre en suivant les saisons, avec un planning établi, s'assurant ainsi une activité stable d'une année sur l'autre. Certains sont même des ouvriers quasipermanents, puisque travaillant quasiment à plein temps avec des contrats saisonniers successifs dans une même entreprise.

Les étudiants constituent une main d'œuvre saisonnière ponctuelle. Pour eux, cette activité est un passage dans leur parcours et représente souvent de leur premier emploi. De manière générale, ils restent sur les sites de production pour une durée n'excédant pas trois mois. La rentrée universitaire se faisant plus tôt, cette catégorie a tendance à diminuer.

La main d'œuvre saisonnière étrangère provient de plus en plus des Pays d'Europe Centrale et Orientale. Depuis le tarissement des sources traditionnelles de recrutement, on les rencontre dans les grandes exploitations qui font appel à un nombre important de salariés saisonniers sur un court moment.

Enfin les «saisonniers incertains » regroupent une population qui n'est pas là par choix et sont généralement orientés chez l'exploitant agricole par un prescripteur. Leurs motivations résident d'abord dans un besoin économique ponctuel ou dans un besoin de pérennisation d'un revenu minimum, mais aussi dans une volonté d'insertion professionnelle. C'est aujourd'hui une catégorie majoritaire dans le paysage saisonnier et ce d'autant plus dans le contexte actuel de crise économique.

\section{Le cas des rosiéristes du Douessin}

\subsection{Design de la recherche}

Cet article s'intéresse à l'expérimentation d'un outil de GTEC - la Charte de l'emploi saisonnier - au sein d'un secteur d'activité et d'un territoire spécifique, à savoir les producteurs de plants de rosiers du bassin Douessin dans le département du Maine et Loire. L'expérimentation de la charte est menée parallèlement dans trois autres territoires. Le choix de ce terrain de recherche résulte d'une opportunité (Girin 1989) offerte par une recherche précédente sur l'institutionnalisation d'une politique RH au sein d'un pôle de compétitivité (Arnaud, et al. 2012).

Notre enquête a, dans un premier temps, consisté à reconstruire la généalogie de l'outil et à identifier les organisations porteur du projet Charte de l'emploi saisonnier. Nous avons ainsi rencontré les deux porteurs de projet au sein de la Chambre d'Agriculture du Maine et Loire et de la Mutuelle Sociale Agricole. Deux autres entretiens ont été réalisés avec le porteur de projet au niveau local au sein de la Maison de l'Emploi de Saumur (données primaires). La première phase de l'expérimentation, menée par les acteurs publics, a consisté à rencontrer six exploitants rosiéristes (données primaires) afin de comprendre l'organisation et la dynamique de la filière ainsi que recenser les pratiques et les besoins en termes de management et de gestion des ressources humaines. En cela, cette recherche constitue une étude multi-cas au sens de Yin (2003).

Au final, quinze entretiens, d'une durée moyenne d'1h30 et intégralement retranscrit, ont été réalisés dont dix avec des entrepreneurs ou DRH d'entreprise du végétal spécialisé. Les cinq autres ont été réalisés avec des membres du pôle. Une réunion organisée par le pôle de compétitivité Vegepolys sur les questions d'emploi et de formation a également été observé. Lors de cette réunion plusieurs parties prenantes au pôle étaient présents (groupements d'employeurs, organismes de formation, DRH, 
dirigeants d'entreprises). La charte de l'emploi est apparue par les professionnels présents comme une démarche adéquate pour améliorer les pratiques RH des entreprises devant déboucher sur une meilleure attractivité de leurs métiers. Enfin, les chercheurs ont eu accès à de nombreux documents (fiches de postes, enquêtes sur les besoins RH des entreprises du secteur, analyse de filière, ...) (données secondaires).

Concernant l'analyse des données, les entretiens retranscrits ainsi que les notes d'observation ont fait l'objet d'un codage manuel et d'une analyse conduite parallèlement par les deux chercheurs participant à l'enquête dans le but d'augmenter la fiabilité de cette dernière. Dans cette recherche exploratoire, nous avons procéder à un codage thématique émergent (Miles et Huberman 1991) quoique partiellement guidé par les items sous-tendant la collecte des données. Parmi ces items prédéfinis, ceux de l'attractivité et de la fidélisation ainsi que les besoins en termes de qualification dans les emplois du végétal ont retenu notre attention. Des thèmes émergents sont par ailleurs clairement apparus durant cette phase comme la difficulté de l'apprentissage de la coopération à de multiples niveaux et l'externalisation d'une compétence clé.

\subsection{La charte de l'emploi saisonnier}

A l'instar de la situation générale du secteur agricole français, la recomposition des relations et l'évolution du travail saisonnier agricole caractérisent le département du Maine et Loire. Les études de l'Association Départementale pour l'Emploi et la Formation en Agriculture (ADEFA) mettent en évidence une double mutation de la structure du travail. Sur les 12 dernières années, on constate d'une part une diminution de la main d'œuvre familiale $(-46 \%)$ et une augmentation de la main d'œuvre salariée $(+34 \%)$ et, d'autre part on assiste à une substitution de la main d'œuvre permanente par une main d'œuvre saisonnière. En Anjou, les activités saisonnières impactent, en 2008, 34.256 salariés, représentant 8.840 équivalents temps plein.

La prise de conscience de cette réalité s'est incarnée dans un programme d'initiative communautaire dénommé Equal «Saisons en Anjou », porteur d'un financement du Fond Social Européen. Mené entre 2001 et 2006, le projet « Saisons en Anjou » a pour objectif d'améliorer la pérennité et la durabilité des emplois saisonniers dans le végétal spécialisé, avec la volonté de prendre en compte tous les aspects depuis la mise en relation avec les employeurs, la formation, les conditions de travail, la garde d'enfants, l'hébergement, la mobilité, la mise en réseau des acteurs. En d'autres termes, il s'agit d'apporter une réponse pluri partenariale à la question de la construction des parcours professionnels des travailleurs saisonniers.

En décembre 2008, dans la continuité de ces travaux et sous l'impulsion du Conseil général du Maine et Loire, a été signée la première «Charte de l'emploi saisonnier ». Adoptée par 38 signataires, la charte de l'emploi saisonnier s'inscrit aujourd'hui dans un « nouveau » projet partenarial porté par la Chambre d'Agriculture de Maine-et-Loire intitulé «Innovation sociale pour l'emploi durable dans le végétal spécialisé », associant la Mutualité Sociale Agricole, le Comité d'Expansion Economique, le Pays des Vallées d'Anjou, le Centre National de Promotion Horticole, Vegepolys et l'ADEFA. Cette charte se veut au cœur de l'innovation sociale, elle symbolise l'engagement de l'ensemble des acteurs dans la prise en compte d'une nouvelle conception de l'emploi saisonnier et a pour ambition de contribuer à une triple reconnaissance.

Il s'agit dans un premier temps de la reconnaissance par l'employeur du rôle des saisonniers dans la dynamique économique de l'entreprise. Les activités saisonnières apparaissent comme l'une des préoccupations majeures des entreprises agricoles. Pris entre l'exigence des marchés d'un côté et l'exigence de la conduite culturale de l'autre, l'emploi saisonnier ne peut plus être considéré comme 
une variable d'ajustement circonstancielle, mais comme une ressource en compétences nécessaires et structurellement disponibles sur un territoire. Il s'agit également de contribuer à la reconnaissance par le salarié de son rôle dans la vie économique et sociale locale. Les offres d'emplois apparaissent comme une nécessité et/ou une opportunité pour ceux qui sont à la recherche d'un emploi. L'emploi saisonnier est caractérisé par l'alternance de deux périodes clés, la saison et la « hors-saison », vécue comme une succession de ruptures entre des périodes d'activité et des périodes sans activité. Il est de la responsabilité de chaque acteur de faire de cette alternance une opportunité en accompagnant le salarié saisonnier pour anticiper, se préparer, capitaliser et construire progressivement un vrai parcours professionnel. Enfin, la charte a pour objectif de renforcer la reconnaissance par les acteurs institutionnels du rôle des activités saisonnières pour la dynamique du territoire. Les tensions résultant de la confrontation entre les besoins des entreprises et les attentes des salariés interrogent sur les missions et les modes de régulation qu'ils mettent en œuvre. Leurs missions sont d'anticiper les besoins en emploi, rendre visible l'offre d'emploi, aider les acteurs économiques dans leur recherche d'emploi ou d'offre d'emploi (guide, formation accueil), accompagner les acteurs dans l'entreprise (accueil, formation de l'encadrement, organisation du travail, prévention sécurité...), consolider la compétence du saisonnier par l'intermédiaire de la formation, développer des services de proximité adaptés (offre en transport, garde d'enfant, hébergement), structurer les règles de l'emploi saisonnier par l'adaptation de la loi. Néanmoins, prises individuellement, aucune organisation ne traite du travail saisonnier dans sa globalité.

\subsection{Le végétal spécialisé, la rose et le bassin du Douessin}

Les productions végétales spécialisées représentent une activité économique majeure de l'Anjou et sont reconnues au sein de Vegepolys, pôle de compétitivité à vocation mondiale du végétal spécialisé. A l'instar de plusieurs autres secteurs d'activités, le végétal fait actuellement face à une crispation de ses marchés se traduisant par une limitation des investissements et des créations d'entreprises. Les acteurs de la filière relèvent également une concurrence accrue, notamment en provenance de l'Europe de l'Est ainsi qu'un mouvement de concentration important en aval de la filière. Pour faire face à la croissance des contraintes externes, les entreprises du végétal sont amenées à s'adapter en industrialisant leur processus de production, en augmentant leur taille, en créant de nouveaux marchés et plus globalement en développant des innovation de produits et de procédés, comme par exemple la création de plants, arbres et fleurs nécessitant moins d'eaux et résistant davantage au froid comme aux grandes chaleurs.

Dans cet environnement turbulent, le rosier reste un produit phare puisqu'il « reste la plante préférée des français », présent dans $77 \%$ des habitats avec jardin ${ }^{3}$ et bénéficiant d'une notoriété en hausse auprès des jeunes et des populations des villes. Avec plus de 10 millions d'unités vendues pour 92 millions d'euros de chiffre d'affaires en 2009 , le rosier représente $2,2 \%$ des volumes de vente de végétaux mais près de $10 \%$ du chiffre d'affaires. En France, la production de rosiers est répartie sur trois territoires : l'Orléanais, le Lyonnais et le Douessin. Chaque bassin d'activité tente, avec plus ou moins de succès, d'innover en développant des variétés plus résistantes mais également en valorisant la qualité de leur production territoriale. Avec 4530000 plants greffés en 2008, le bassin Douessin se situe en tête de ses trois territoires et représente près de $45 \%$ de la production nationale.

Les rosiéristes du bassin Douessin sont constitués d'environ 80 exploitations réparties en trois grandes familles. Les exploitations dites «commerciales» $(15 \%)$, qui produisent et vendent leur production auprès de la clientèle. Certaines de ces exploitations ont «pignon sur rue » depuis plusieurs

\footnotetext{
${ }^{3}$ Baromètre « Les français et le Végétal » - automne 2009 :

http://www.snhf.org/images/stories/2_Marche horticole/barometre6.pdf
} 
générations. La seconde famille regroupe les exploitants «contractants » (70\%), qui reçoivent une commande auprès d'un faiseur d'ordre (les faiseurs de rosiers) pour greffer une certaine quantité et qualité de rosiers. La démarche commerciale est essentiellement contractuelle et ces exploitants n'ont pas de clients «physiques ». Enfin les exploitations dites «semi commerciales» (15\%) combinent les deux formes précédentes de commercialisation.

Le Douessin se caractérise par une production atomisée, diminuant classiquement leur rapport de force vis-à-vis de leur clients concentrés (Grande Distribution Généraliste et Spécialisée). Cette situation ne permet pas de développer des marges suffisantes pour organiser l'industrialisation de la filière locale : concentration, mécanisation, innovation produit, communication (produits, labels, emplois), enquêtes clients, calculs de prix de fabrication et de revient ... et attraction et fidélisation des compétences de plus en plus rares. Egalement, les acteurs de la filière semblent peu enclins à entrer dans des stratégies de type collective afin d'organiser la mutualisation des ressources et gagner en taille critique : une coopérative créée en 2001 par les sous-traitants devait fermer au moment de l'enquête début 2011 ; des entrepreneurs occupés quotidiennement par la production et disposant de peu de temps pour s'engager dans des mouvements collectifs.

Pour autant, ces rosiéristes sont situées au sein d'une région de production horticole et pépinière importante. En effet, l'horticulture constitue l'une des principales activités des territoires limitrophes au Douessin (pour faire simple le département du Maine et Loire) : 90\% de la production destinés aux marchés, local et national, cultivés sur 3400 hectares ; 10\% de la production exportés, surtout en Europe. Le territoire est au $1^{\mathrm{er}}$ rang national pour les plantes en pots, les plantes à massif, les jeunes plants de pépinière, les bulbes, le dahlia, les arbustes en conteneur. Enfin, 280 entreprises sont présentes et regroupent plus de 3300 emplois permanents. On notera que compte tenu du caractère saisonnier de l'activité, la saison de haute activité représente une multiplication par 5 à 25 du nombre de salariés au sein des organisations concernées.

Dans cette situation, les leviers stratégiques et organisationnels des acteurs de la filière s'orientent vers un renforcement des collaborations entre exploitants du Douessin ainsi qu'avec les autres secteurs du végétal du département se matérialisant par une stratégie de communication et de labellisation visant à attirer, fidéliser et mutualiser des ressources humaines qualifiées. Les collectivités territoriales se sont alors lancées dans la rédaction d'une charte de l'emploi saisonnier, relayée et expérimentée par la chambre d'agriculture, la MSA et les maisons de l'emploi.

\subsection{L'expérimentation de la charte de l'emploi saisonnier chez les rosiéristes du Douessin}

A la lumière des entretiens menés, il apparaît que l'expérimentation de la Charte de l'emploi saisonnier contribue à une meilleure connaissance des besoins des exploitants rosiéristes. Cela étant, cette connaissance émergente a pris des détours insoupçonnés.

Au premier abord, deux problématiques initiales ressortent des entretiens menés auprès des exploitants rosiéristes en termes d'attraction et de fidélisation des salariés saisonniers. Ces problématiques correspondent à deux périodes d'activité distinctes, la période d'arrachage et la période de greffage ou d'écussonnage. La période d'arrachage se situe entre le 15 septembre et le 15 décembre. Les difficultés de recrutement de saisonniers en période d'arrachage sont réelles. Les conditions de travail sont particulièrement éprouvantes au regard des conditions météorologiques et une posture de travail courbé.

La période de greffage débute généralement le 15 juillet et s'achève le 30 août. La problématique de fidélisation en période d'écussonnage n'a pas été évoquée par les rosiéristes lors des entretiens. En effet, il ressort des entretiens l'émergence et l'utilisation d'une main d'œuvre étrangère européenne, 
principalement anglaise. Cette catégorie de saisonniers connaît un essor certain auprès des exploitants rosiéristes. Les entretiens montrent que plus de 50\% des greffes sont effectuées par les anglais, même si ce chiffre est difficilement vérifiable. Plusieurs éléments ont été soulevés par les répondants. Premier point, les travailleurs saisonniers anglais vendent une prestation de service et sont payés à la tâche pour un coût compris entre 17 cts et 21 centimes du pied. Or le paiement au pied est interdit par la législation française. De plus ces saisonniers ne sont pas soumis aux mêmes contraintes horaire et journalière du travail :

«Ce sont des gens qui n'ont pas de soucis d'horaires. Alors que nous, avec nos jeunes, c'est tout à fait légitime mais pour les 14-15 ans, c'est 32 heures, pour les 16 ans c'est 35h... Alors déjà quand on compose une équipe, vous avez 2 greffeurs, 1 enveloppeur, si vous n'avez pas les mêmes horaires, ça complique les choses. Et puis la législation française, elle est celle-ci : il pleut pendant 1 jour ou 2 dans la semaine, vous ne pouvez pas greffer, j'ai posé la question à l'inspection du travail, même si je connaissais déjà la réponse. "monsieur l'inspecteur, il a plu, il faudrait qu'on puisse récupérer le samedi matin", "surtout pas! Que je ne vous vois pas travailler samedi matin!". Et on le fait quand le travail? En clair, ça c'est notre problème et nous ne nous sentons pas aidés... »

De surcroit, ces travailleurs n'ont pas besoin d'encadrement, ils connaissent la spécificité du métier et font preuve d'une réelle autonomie: «les anglais viennent chercher les greffes dans le frigo et $s$ 'autogèrent ». A contrario, l'utilisation d'une main d'œuvre française nécessite un encadrant pour vingt jeunes saisonniers.

Enfin, en termes de productivité, deux greffeurs anglais peuvent produire jusqu'à 3000 pieds par jour à comparer avec la production moyenne d'un greffeur français, c'est-à-dire aux alentours de 1000 pieds par jour. Il apparaît, pour expliquer ce différentiel, qu'à côté de la technique traditionnelle de greffage utilisée par les exploitants rosiéristes, la technique de greffage anglaise comporte un avantage certain de fiabilité : $100 \%$ de réussite contre $90 \%$ pour la greffe française :

«En termes de rendement il n'y a pas photo. A deux ils greffent et enveloppent au minimum 2500 rosiers par jours, nous pour faire autant, il nous faut au minimum quatre personnes ».

Il est à remarquer que les risques liés par l'utilisation de cette main d'œuvre étrangère sont largement reconnus par les exploitants, notamment en termes de perte de savoir-faire, de dépendance au prix et vis-à-vis du rôle social au regard de la population locale :

«Des saisonniers anglais, il en vient même de plus en plus. C'est chaud, mais c'est la réalité, il ne faut pas se voiler la face. Mais je le dis, attention... attention parce que faut pas perdre notre savoir-faire, on a un rôle social, nos jeunes sont bien mieux à bosser plutôt qu'a traîner. Il faut qu'on soit extrêmement vigilent. Mais d'un autre côté vous avez un certain nombre de contraintes qui s'amplifient... Ça rend bien service quand même! Même si les salaires partent en Angleterre, et les charges également partent en Angleterre ».

Le second aspect concerne des besoins en termes de mutualisation des pratiques même si le président de l'association des rosiéristes relève aussi le manque de solidarité qui existe entre les pépiniéristes. L'importance de trouver "un intérêt personnel dans une démarche collective » apparaît comme un enjeu majeur de la dynamique territoriale et organisationnelle.

Une première illustration concerne la nécessité de construire une solution locale à la problématique des parcours professionnels des travailleurs saisonniers :

"Le complément d'activités peut être la solution. Moi je le pense. Il faudrait qu'on puisse arriver à s'organiser pour que les saisonniers motivés ait une rémunération plus convenable qu'un saisonnier "qui vient boucher un trou" et qu'on arrive à s'organiser pour 
qu'ils aient des propositions de travail dans d'autres domaines qui leur permettent d'avoir une activité toute l'année. Il y a bien la solution des groupements d'employeurs, mais c'est plus onéreux! Forcément. Entre un occasionnel embauché par notre entreprise et un occasionnel embauché par un groupement d'employeurs...C'est minimum $4 €$ de l'heure de différence ».

Au-delà de cette problématique, les possibilités d'interaction au niveau du territoire sont réelles :

"Un chantier que nous avons lancé, c'est le marché aux végétaux Pépifolies à Doué la Fontaine tous les mois de novembre. Avec tout ce potentiel de pépiniéristes avec ces fêtes de la rose depuis 51 ans...paradoxalement, il n'y avait pas de marché aux végétaux. Donc nous avons mis en place le marché aux végétaux qui a permis à une bonne vingtaine de pépiniériste de se retrouver pendant 48 heures sur le même lieu. Entrée, parking gratuit. Nous avons eu la première année environ 1500 personnes avec un temps exécrables, 2ème année, là on a eu pratiquement 4000 personnes venant de 9 départements. Et l'année dernière pour la 3ème année nous en avons eu environ 5000 venants de 31 départements. Donc là, la mayonnaise commence à prendre. L'intérêt de ce marché aux végétaux, tout d'abord, c'est de permettre aux entreprises, quelle que soit leur dimension et leur production de se retrouver sur le même site, et puis bien sur l'organiser au mieux. . On va essayer pour la 4ème édition de l'ouvrir à d'autres professions horticoles et pour ce faire nous sommes soutenus par la municipalité, la commune, le conseil régional et bon d'acteurs institutionnels ».

Cela étant, l'histoire de la coopérative des rosiéristes laisse à penser que cette mutualisation ne va pas de soi. Composée de 49 adhérents, la coopérative a connu deux dépôts de bilan en 2003 et 2006 et est en cours de fermeture définitive. De l'avis du président de l'association des rosiéristes, plusieurs éléments ont concouru à cette situation. D'une part, il n'y avait pas de prix de base, aucune de valeur ajoutée et surcout de $10 \%$. D'autre part, elle n'a pas été favorisée par les grosses entreprises du bassin, ce qui explique qu'elle ne pesait pas dans la négociation.

\section{Discussion}

L'objectif de cette recherche était d'étudier la mise en place et les répercussions d'un outil de GTEC la Charte de l'emploi saisonnier - au sein du bassin du Douessin, première région française de production de rosiers. Cette charte a été conçue comme un moyen devant permettre aux exploitations agricoles de faire face aux problématiques annoncées d'attractivité et fidélisation de la main d'œuvre saisonnière. L'analyse de ce cas contribue à la compréhension du phénomène actuel de territorialisation des pratiques RH comme réponse aux problématiques contemporaines d'emploi et plus spécifiquement de l'emploi saisonnier. Nous discutons maintenant les nos implications théoriques et managériales.

Ces constats et implications issus du terrain permettent par ailleurs d'élargir la discussion aux travaux croissants en GRH territoriale. Tout d'abord, les situations présentées dans cet article démontre, et confirme, la multiplicité des acteurs concernés et des formes de gestion territoriale des ressources humaines possible fonction des problématiques locales rencontrées. Par ailleurs, la difficulté de recruter n'est pas apparue si évidente que cela à la différence des observations de Glee et Roger (2007) dans le BTP et la restauration. Les exploitants rosiéristes fonctionnent pour une part avec une main d'œuvre de proximité, fidélisée années après années essentiellement pour les saisons hors été et ont fait le choix volontaire d'externaliser à une main d'œuvre étrangère, tout comme les secteurs observés par Glee et Roger (2007), une compétence clé de leur métier : le greffage. Dans notre cas, cette décision ne s'est pas tant imposée faute de mieux que compte tenu des avantages organisationnelles qu'elle donnait (pas besoin de surveillance, déjà formée, productivité et qualité supérieure). En cela, il s'agit d'un décision délibérée tendant progressivement à se généraliser au risque de voir les rosiéristes pieds et mains liés au 
bon vouloir de cette main d'œuvre, certes efficace et rentable, mais tout de même potentiellement volatile et susceptible de multiplier par deux ou trois leurs tarifs une fois la dépendance pleinement réalisée. Cette situation, à laquelle il est possible d'ajouter l'échec de la coopérative, illustre la difficulté de ces exploitants à coordonner leurs efforts, au sein de la profession mais aussi avec les organismes extérieurs, afin de faire valoir leurs intérêts collectifs. Ce point illustre également le faible degré d'interdépendance perçu par les rosiéristes, constituant pourtant l'une des conditions de réussite d'une GRH territorialisée (Pichault 2010). Enfin, comme Defelix et al. (2010) l'ont montré, la gestion territoriale des ressources humaines, portée par des organismes publics, s'inscrit régulièrement dans une logique de redynamisation d'un bassin d'emploi poursuivant in fine un objectif à deux niveaux. A minima, il s'agit de sauvegarder l'existant dans le but d'éviter la quasi-disparition des formes d'emploi sur un territoire donné. De manière plus positive, l'objectif est de remobiliser, en les réorganisant, les forces vives du territoire concerné autour d'un ou plusieurs secteurs d'activités afin de lui redonner de l'attraction et ainsi pouvoir imaginer un cercle vertueux de développement (économique, démographique, social, etc.) à venir.

Au final, c'est la question de l'apprentissage à divers niveaux qui est en jeu. En cela, notre article confirme les résultats de Bories et Loubès (2009) sur l'importance et la difficulté de l'apprentissage dans les démarche GTEC portées par les maisons de l'emploi. Ces auteurs considèrent en effet que le développement de toute GTEC passe par un triple apprentissage: de la coopération des acteurs impliqués et concernés par le projet; de la définition du territoire d'action; et enfin de la gouvernance à l'origine du projet de gestion territoriale des ressources humaines. Concernant la définition du territoire, comme précisé en section méthodologique, l'expérimentation de la charte de l'emploi saisonnier a été réalisée dans 3 sous-territoires géographiquement distincts et avec des activités différentes (horticulture dans notre cas, champignon, cidriculture). Cette première étape a permis une définition précise des territoires qui ont, à la différence du cas étudié par Bories et Loubès, la caractéristique d'être peuplé de saisonniers ancrés géographiquement (en dehors des anglais dans notre cas). Ce point a clairement facilité le premier travail d'expérimentation. Lors de la généralisation à venir de l'outil, les frontières risquent en revanche d'être plus poreuses et sources de difficultés. Ensuite, le projet Charte constitue une volonté du CG 49 opérationnalisée par la MSA 49 et l'ADEFA. L'expérimentation a été menée en direct par deux représentantes de chacune de ces institutions pour deux des trois secteurs identifiés. Le troisième territoire ayant été confié à une personne de la MDE de Douessin, cas étudié ici. Cette division spécifique de la gouvernance a rendu possible l'expérimentation. La généralisation du projet charte devra en revanche soulevé le double défi de l'harmonisation des pratiques entre institutions et le remplacement de la porteuse du projet dans le Douessin ayant quitté ses fonctions depuis. Enfin, les dimensions «implication des acteurs et volonté d'apprendre à travailler ensemble» posent véritablement problème à deux niveaux: institutionnel et organisationnel. Dans notre cas, la charte a été expérimentée par la MDE du Douessin. Sur ce point, et de manière classique (Bories-Azeau et Loubes 2009), les MDE sont en concurrence avec Pôle Emploi dont elles dépendent, et impliquent une coopération potentiellement délicate. Par ailleurs, les acteurs des TPE concernés rencontrent visiblement des difficultés à s'impliquer dans des démarches collectives (cf. cas de la coopérative), situation d'autant plus paradoxale que les conditions de succès de l'implémentation de la charte et du développement voire de la pérennité de leurs exploitations en dépendent.

En second lieu, les praticiens de la RH territoriale - métier RH en réel essor - se retrouveront certainement dans cette recherche à travers les défis RH rencontrés mais aussi dans les quatre préconisations managériales qui en découlent. L'auto diagnostic de la démarche Charte de l'emploi saisonnier a permis et facilité l'interaction et l'action collective au niveau d'un territoire de manière à la fois délibéré et émergente. En effet, alors que la démarche était initialement destinée à résoudre des problématiques liées au recrutement et à la fidélisation du personnel saisonnier, le bilan intermédiaire 
de cette expérimentation permet de relever d'autres questionnements comme la réflexion en termes de stratégie collective, la formation au greffage afin de diminuer le risque stratégique d'une perte de savoir-faire ou encore la mise en règle par rapport au droit sanitaire et du travail, autrement dit à une mise en mouvement générale des acteurs de la filière. Ce point est particulièrement saillant au regard des préconisations issues de ces entretiens. En effet, aucun des rosiéristes rencontrés n'a fait part de difficultés à attirer et fidéliser les saisonniers pour le greffage. Pourtant, devant le constat d'un recrutement de plus en plus important des greffeurs anglais, alors qu'aucun saisonnier français n'est formé à la greffe anglaise sur le territoire, et que les rosiéristes eux même ne maitrisent pas ce mode de greffage, l'idée selon laquelle il est utile de proposer aux rosiéristes d'expérimenter une formation à la greffe anglaise pour les saisonniers prend son sens. Avant de proposer cette formation, une phase préparatoire et une étude de faisabilité du projet doit être menée. A savoir, trouver un formateur compétent en greffe anglaise disponible et trouver des financements. L'idée serait également de conserver une comparaison avec les greffeurs anglais chez un même rosiériste. L'accompagnement sur le coût de production en amont et pendant l'expérimentation serait souhaitable.

La seconde préconisation concerne la problématique du temps de travail partagé. Il s'agit alors d'identifier les activités proches dans des secteurs à saisonnalité complémentaire. En effet, la problématique de fidélisation des saisonniers est liée directement au fait d'une recherche d'emploi à temps complet. Des recherches ont été effectuées par les différentes structures pour étudier les complémentarités. Dans le cas présent, il semble que seule la viticulture soit compatible avec un temps partagé à l'année. Les entreprises de l'industrie sont difficiles à solliciter car elles utilisent un personnel intérimaire et aucune démarche n'a été effectuée auprès des agences d'intérim.

Le troisième point rend compte de la méconnaissance des rosiéristes rencontrés au niveau des services qu'ils peuvent utiliser pour recruter, et particulièrement les saisonniers. A cette fin, il est préconisé de communiquer via la création d'une plaquette territoriale regroupant l'offre de service des partenaires pouvant accompagner les employeurs. Ce projet a déjà été initié sur un autre territoire du département. La Maison de l'Emploi propose à ce titre de compiler les informations auprès de groupements d'employeurs pour réfléchir sur l'accompagnement possible pour le recrutement des saisonniers.

Enfin la problématique de la santé et de la sécurité au travail semble être des thématiques prégnantes dans la filière en raison des positions courbées, ou en force. Parmi les préconisations, il est évoqué de proposer à la Mutuelle Sociale Agricole d'intervenir sur cette thématique auprès des entreprises volontaires.

\section{Conclusion}

L'objectif de cet article était d'étudier l'expérimentation d'une charte de l'emploi saisonnier dans le bassin du Douessin. La dimension GTEC de cet outil apparait clairement. Cette recherche confirme la nécessité d'un apprentissage au carrefour du territoire et des branches d'activités (Bories-Azeau et Loubes 2009), et met en avant des enjeux autant stratégiques que RH, autour de la perte d'une compétence cœur. En cela, l'un des défis majeurs de nos rosiéristes reste de s'appuyer sur leur proximité géographique pour développer des formes de proximité organisationnelle (des processus et pratiques rapprochées) et institutionnelle comme les y invite Zimmerman (2008). L'expérimentation de la charte a été utilisée comme un outil facilitant l'interaction et l'action collective au niveau d'un territoire (création et lancement prochain de formation au « greffage à l'anglaise » par Pôle Emploi et la région), plus éloigné d'un réel maillage de compétences (Bories-Azeau, et al. 2012, à paraitre) ayant pu contribuer à la résolution du problème de rareté de la main d'œuvre et de valeur du territoire. En cela, il 
est important de souligner que toute démarche GTEC ne doit et ne peut se limiter à un ou des outils aussi intéressants soient-ils. En effet, ce que montre cette recherche, c'est qu'une politique territorialisée de gestion des ressources humaines n'est rien de plus qu'un moyen au service d'une stratégie d'un territoire. C'est certainement sur ce point qu'il est possible de relier pratiques RH traditionnelles et actions GTEC. Or, c'est bien là que réside la difficulté des territoires : formuler et opérationnaliser une stratégie globale traduisible en stratégie et politiques RH. 


\section{Annexe 1 : extrait de la charte de l'emploi saisonnier Source : http://maine-et-loire.anefa.org/emploi-1}

\section{La saison : Rassembler les conditions pour réussir le travail}

Article 1 : Accueillir dans l'entreprise

Je suis employeur

- J'informe dès que possible des dates de démarrage et de fin de saison.

- Je rencontre le salarié saisonnier, si possible avant le démarrage de la saison.

- Je lui fais visiter l'entreprise, lui explique son rôle dans le cycle de production et lui présente le contenu du poste ; je lui donne un livret d'accueil.

- J'explique au salarié saisonnier le contenu de son travail, à partir d'une description écrite des tâches à réaliser ou d'une fiche de poste.

- J'implique les salariés permanents dans l'accueil des saisonniers.

- Je propose des lieux de vie (lieu de repas, vestiaire, sanitaires, parking).

\section{Article 2 : Organiser le travail ; promouvoir la sécurité au travail; respecter les consignes}

Je suis employeur

- J'identifie et je communique sur mes besoins de main-d'oeuvre.

- J'aménage les postes de travail et fournis les équipements appropriés pour de meilleures conditions de santé et de sécurité.

- J'informe le salarié saisonnier sur les règles de sécurité liées à son environnement de travail et nécessaires à la tenue de son poste.

- Je présente au salarié saisonnier ses référents au sein de l'entreprise.

- J'adapte les horaires, quand c'est possible, pour lever les contraintes d'organisation des salariés (horaires de bus, garde d'enfants...).

Je suis acteur de l'emploi, de la santé et sécurité au travail

Pôle Emploi, DDTEFP, missions locales, PAIO, PLIE, associations intermédiaires, ADEFA, Chambre

d'Agriculture,

MSA...

- J'oriente et j'accompagne les salariés saisonniers dans leur recherche d'emploi.

- J'accompagne l'employeur dans le recrutement des salariés saisonniers.

- J'informe l'employeur sur les services proposés et l'accompagne pour améliorer la prévention des risques dans l'entreprise.

J'informe l'employeur sur les services proposés et l'accompagne pour améliorer les relations humaines et l'organisation du travail.
Je suis salarié saisonnier

- Je prends des dispositions pour régler les questions liées à la garde de mes enfants, ma mobilité, mon hébergement. Je prépare mon équipement de travail.

- Je suis présent tous les jours de travail, je suis ponctuel et je préviens dès que possible en cas d'empêchement.

- Je demande des explications sur les tâches à effectuer dès que je ressens une difficulté.

- J'éteins mon téléphone portable pendant les heures de travail.

- Je tiens compte des recommandations sur la tenue vestimentaire la plus adaptée.

- Je respecte les consignes de sécurité, pour moi-même et les autres.

- Je prends un petit déjeuner et je m'hydrate.

- Je respecte les lieux mis à ma disposition.

- Je m'adapte à l'organisation de l'entreprise

- Je fais part de mes contraintes d'organisation personnelle.

Ensemble, employeur et salarié saisonnier

- Nous faisons, en entretien individuel, un bilan du travail réalisé pendant la saison.

- Nous nous informons mutuellement de notre souhait de travailler ensemble à nouveau la saison prochaine. 
Je suis employeur

- J'utilise la période d'essai pour accompagner le salarié dans sa progression vers les objectifs fixés.

- Je forme les encadrants aux relations humaines.

- A la fin de la saison, j'informe le salarié saisonnier sur les dispositifs de formation existant.

Ensemble, employeur et salarié saisonnier

- A la fin de la saison, nous mettons en évidence, par écrit de préférence, les compétences acquises et mises en oeuvre pendant la saison et celles qu'il serait utile d'acquérir.
Je suis acteur de l'emploi, de l'insertion et de la formation

Pôle Emploi, DDTEFP, missions locales, PAIO, PLIE, associations intermédiaires, ADEFA, FAFSEA, centres de formation...

- J'informe l'employeur sur les dispositifs de formation existant et l'accompagne dans leur mise en oeuvre.

\section{Article 4 : Connaître ses droits et devoirs}

Je suis employeur

- Je me forme au droit du travail pour mieux connâ̂tre mes droits en tant qu'employeur et mes devoirs envers mes salariés.

Je suis salarié saisonnier
- Je m'informe sur mes droits en tant que salarié, mes devoirs envers mon employeur et mes obligations envers les institutions.

\section{Entre les saisons : Gérer l'alternance pour mieux vivre les saisons} Article 5 : Accompagner vers l'emploi

Je suis salarié saisonnier

- J'informe de la fin de mon contrat et fais valoir mes droits à l'emploi.

Je suis acteur de l'emploi et de l'insertion

Pôle Emploi, DDTEFP, missions locales, PAIO, PLIE, associations intermédiaires, ADEFA...

- J'informe les salariés saisonniers sur les dispositifs d'accompagnement pour rechercher un emploi ou réaliser un projet.

- J'accompagne les salariés saisonniers dans la réflexion sur leur parcours professionnel en valorisant les compétences acquises pendant la saison.

- Je favorise l'information des employeurs sur le droit du travail applicable à l'emploi saisonnier.
Je suis acteur du développement et de l'organisation du territoire

Conseil Général, collectivités, Pays, DDTEFP, MSA, missions locales, PAIO, PLIE...

- Je favorise l'accès aux services facilitant l'emploi (mobilité, garde d'enfants, hébergement...) en informant, en adaptant les conditions d'accès, en améliorant leur souplesse.

- Je favorise la création de structures de type groupements d'employeurs pour aider les salariés saisonniers à accéder au temps plein annuel.

- Je soutiens les initiatives collectives en faveur de l'accueil des salariés saisonniers (logement, sanitaires, matériel...). 
Je suis salarié saisonnier

- Je conforte mon parcours professionnel en faisant valoir mes droits à la formation.
Je suis acteur de l'emploi, de l'insertion et de la formation

Pôle Emploi, DDTEFP, missions locales, PAIO,

PLIE, associations intermédiaires, ADEFA,

FAFSEA, centres de formation

- J'informe les salariés saisonniers sur les droits et les

conditions d'accès à la formation.

\section{Article 7 : Accéder aux soins}

Je suis salarié saisonnier

- Je m'assure de mes aptitudes physiques en faisant valoir mes droits à la santé.

Je suis acteur de la promotion de la santé

Associations, MSA...

- J'informe sur les droits à la santé, je facilite l'accès aux soins, je développe des actions de prévention.
Je suis acteur du développement et de l'organisation du territoire

Conseil Général, collectivités, Pays, MSA,...

- Je contribue à une meilleure organisation pour faciliter l'accès aux soins sur le territoire.

\section{Article 8 : Promouvoir les activités saisonnières et la charte de l'emploi saisonnier}


Je suis employeur

- Je participe à un réseau d'échange de bonnes pratiques sur le travail saisonnier.

Ensemble, acteurs de l'emploi, de la formation, de l'insertion, du développement et de l'organisation du territoire, de la promotion de la santé

- Nous participons à un réseau d'échanges sur l'emploi saisonnier.

- Nous participons à la promotion et à la diffusion de la charte de l'emploi saisonnier.

\section{Références}

Arnaud N. (2007), "Construction et management de compétences collectives dans le cadre de relations interorganisationnelles : une approche communicationnelle. Le cas du secteur du transport de meubles neufs en France ", Thèse de doctorat en Sciences de Gestion, Université de Nantes, novembre.

Arnaud N., Fauvy, S. et Nekka, H. (2012), " La difficile institutionnalisation d'une politique de GRH au sein d'un pôle de compétitivité : Une étude de cas exploratoire ", Revue française de gestion, n`à paraitre,

Barabel M., Chabault, D., Meier, O. et Tixier, J. (2009), " La dynamique de territoire et l'évolution d'un pôle de compétitivité: le cas de Cosmetic Valley ", Management \& Avenir, n5, 25, p.144-163.

Bories-Azeau I., Faillenet, P., Loubès, A. et Commeiras, N. (2012, à paraitre), " GPEC et logique territoriale ? Un exemple dans l'industrie ferroviaire ", in R. Soparnot et D. Leroy (Ed.), La gestion des compétences : les nouveaux territoires,

Bories-Azeau I. et Loubes, A. (2009), « Les maisons de l'emploi, un dispositif en faveur d'une gestion territoriale des ressources humaines ? ", XX⿳亠口冋 Conférence de l'AGRH, Toulouse.

Bories-Azeau I., Loubès, A. et Fabre, C. (2007), « Emergence d'un acteur collectif territorial et réseau d'entreprises: I'exemple de CAMDIB ", Revue Gestion et Management Publics,

Calamel L., Defélix, C., Mazzilli, I. et Retour, D. (2011), " Les pôles de compétitivité: un point de rupture pour la GRH traditionnelle? Une analyse des dispositifs $\mathrm{RH}$ au sein des 12 pôles de la région Rhône-Alpes ", Management \& Avenir, $n^{\circ} 1,41$, p.175-193.

Colle R., Culié, J.-D., Defélix, C., Hatt, F. et Rapiau, M.-T. (2009), "Quelle GRH pour les pôles de compétitivité ? ", Revue Française de Gestion, p.143-161.

Defélix C., Colle, R. et Rapiau, M.-T. (2009), " Prendre en compte le facteur humain au sein des pôles de compétitivité : la longue marche vers l'innovation sociale. ", Revue Management et Avenir, p.9-29. Defélix C., Colle, R. et Rapiau, M. (2007), " Les pôles de compétitivité: un creuset pour l'innovation sociale ", Congrès de l'AGRH,

Defélix C., Dégruel, M., Le Boulaire, M. et Retour, D. (2010), "Territorialisation de la GRH : de nouvelles démarches d'entreprise et une nouvelle GRH ? ", XX Conférence de l'AGRH, Saint-Malo, France.

Girin J. (1989), "L'opportunisme méthodique dans les recherches sur la gestion des organisations ", La recherche en gestion, Paris.

Glee C. et Roger, A. (2007), " Attraction et fidélisation de la main d'oeuvre dans le secteur du BTP et de l'hotellerie-restauration : le paradoxe de la rareté ", XVII ${ }^{\circ}$ Congrès de l'AGRH, Fribourg, Suisse.

Guais G. (2007), La durabilité de l'emploi agricole saisonnier en question : le cas du Baugeois-Vallée, Mazzilli I. (2009), "Une Gestion des ressources humaines à l'interface des organisations: vers une GRH territoriale? ", AGRH, Toulouse.

Mazzilli I. (2010), " GRH, Territoire et Traduction, ou la difficile naissance d'une gestion territoriale des ressources humaines ", XX Conférence de I'AGRH, Saint-Malo, France.

Mendras H. (1970), La fin des paysans: changement et innovations dans les sociétés rurales françaises, Armand Colin.

Miles M. B. et Huberman, A. M. (1991), Analyse des données qualitatives : recueil de nouvelles méthodes, De Boeck. 
Pichault F. (2010), " intervention au groupe de recherche thématique " GRH et territoire » ", Paris, février 2010,

Raulet-Croset N. (2008), "La dimension territoriale des situations de gestion ", Revue française de gestion, 4, p.137-150.

Yin R. K. (2003), Case Study Research, Design and Methods, Sage.

Zimmermann J. B. (2008), "Le territoire dans l'analyse économique ", Revue française de gestion, 4, p.105-118. 